\title{
GRAY TONE FILTERING ON ERS-SAR IMAGES APPLIED TO CHANGE DETECTION AND MAPPING
}

\author{
GILLES ANDRÉ1,2, Sigrid HeSS ${ }^{1}$ AND CATHERINE MERING ${ }^{1}$
}

${ }^{1}$ UMR CNRS PRODIG, Université Paris 7 Denis Diderot, 2, place Jussieu, F 75252 Paris Cedex 05; ${ }^{2}$ Geosciences Consultants, 157 rue des Blains 92220, Bagneux, France

e-mail: gilles_p_andre@yahoo.fr; sigrid@univ-paris-1.fr; mering@lgs.jussieu.fr (Accepted September 30, 2002)

\begin{abstract}
In SAR images, the pixel values are tightly related to physical parameters of the soil such as topography, roughness and humidity, regardless to atmospheric conditions. Therefore, SAR images may be used to detect, and quantify changes in land cover, by comparison of time series SAR data. Classical change detection techniques from SAR images are based on additive synthesis of RGB colors and arithmetic operations between images. The noisy aspect of ERS image due to the original speckle is an obstacle for available mapping and quantification of the changes. Here, statistical and morphological filters are used to reduce the speckle noise. Combined techniques of change detection and noise filtering are applied here to assess and map from ERS-SAR images the impact of regular or catastrophic flood and deforestation in the East Coast of Madagascar.
\end{abstract}

Keywords: change detection, filters, remote sensing, SAR image.

\section{INTRODUCTION}

Due to their high degree of repetitivity, and an acquisition practicable during night and cloudy weather, SAR (synthetic aperture radar) images from the ERS satellite are often more available than optical images for the mapping of changes in any meteorological conditions.

Yet, SAR images include a speckle noise impeding digital processing. Speckle noise is due to that every pixel in a SAR image carries an integrated information i.e., the sum of the elementary reflections of multiple objects contained in SAR image resolution cell. In this respect, SAR images do not contain regions with a spatially uniform reflectivity: pixels of a given gray level are generally not contiguous. Therefore, the classical methods for feature extraction cannot provide the satisfactory results achieved with optical images. To improve the change detection from multi-temporal ERS data, it is necessary to reduce the speckle noise in the original images by gray tone filtering.

In this work, we experiment two kinds of filtering method to assess, and map the impact of flood and deforestation on a multi-temporal series of SAR images. The two methods are compared to another filter, the so-called Lee filter, commonly used to reduce noise in SAR images. Results are presented for two case studies. In the first example, we rely on ERS images of the Northeast Coast of Madagascar (Maroentsetra) to map the impact of flood due to the three hurricanes of February and March 2000. The second example shows the impact of deforestation from 1995 to 2000 in the north of Madagascar (Antalaha).

\section{CHANGE DETECTION}

Many methods like color composition, ratio (Singh, 1989) or PCA (Gong, 1993) have been developed to detect changes between two or more ERS SAR images.

The first and most common approach consists in making a false color composition with two images: the red and blue channels are the image of the first date and the green the second one. This technique is used to show an increase or a decrease of backscattering between the two images. A high increase of backscattering would appear in green and a decrease in magenta. We used this approach to detect both the surfaces affected by flood due to the hurricanes of February and march 2000 in Madagascar (Fig. 1) and the area affected by deforestation in the North of Madagascar (Fig. 2). In Fig. 1, the increase of backscattering (in green) may be attributed to a very wet sedimentary deposit while the decrease (in magenta) expresses the presence of water. In Fig. 2, the method allows the detection of the deforested area characterized by a very high decrease of backscattering due to a reduction of the roughness. 


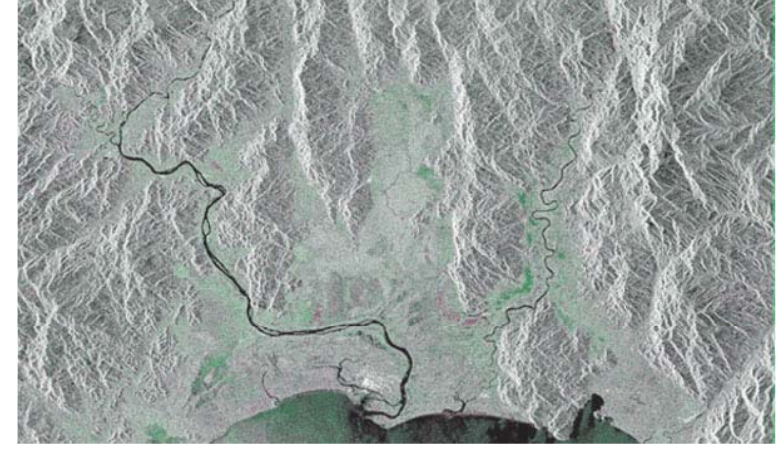

Fig. 1. False color composition of two ERS images of the Maroentsetra delta. In Red and blue: the ERS SAR image of April 1995; in Green the ERS SAR image of April 2000. We see in green the area of wet sediment deposit yielding an increase of backscattering, and in magenta the flooded area characterized by a decrease of backscattering.

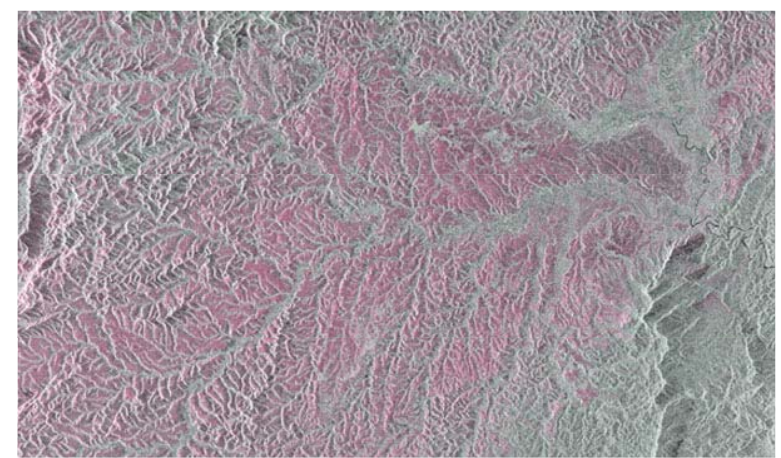

Fig. 2. False color composition (RGB) of the deforested area of Antalaha In blue and red: the image of April 1995; in green the image of April 2000. The magenta color is the area of deforestation.

A second type of method is based on the computation of a ratio or a difference between images highlighting variations of backscattering (BS) interpreted as significant changes of the surface state. This class of methods are applied to two ERS-SAR images of Madagascar (Figs. 3 and 4). In both Figs. 3 and 4, a dark gray value expresses an increase of BS and a light gray value shows a decrease of BS .

The last type of method considered herein is Principal Component Analysis. This technique enhances the contrast of BS between time series images by decorrelating the images. Usually the first component is associated to a maximum of variance and is interpreted as the invariant across the images. The other components express the residual variance associated to changes. We applied this technique to the two ERS SAR images and the image of ratio to show the deforestation (Fig. 5) and flooded area in Madagascar (Fig. 6). Changes, concentrated on the second and third components, appear in a yellowgreen tone in the false color composition (Fig. 6) by addition of green (component no. 2) and red (component no. 3).

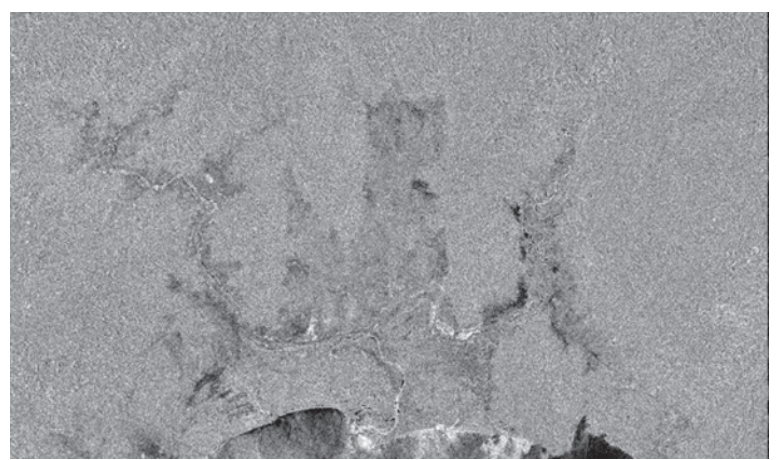

Fig. 3. Image Ratio of Maroentsetra delta calculated from two ERS SAR Images. Black pixels show an increase of backscattering (BS) (wet sediment deposit); white pixels show a decrease of BS (flooded area). The middle gray levels express the stability of $B S$ in the two images.

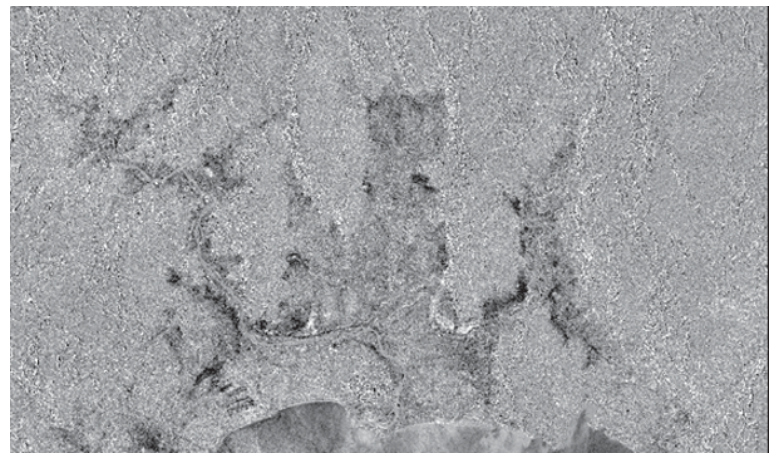

Fig. 4. Image of difference between two ERS images of Maroentsetra delta. Dark gray values show an increase of $B S$ and light gray values a decrease of $B S$. This result is quiet close to the image ratio result (Fig. 3) but includes a more noisy aspect.

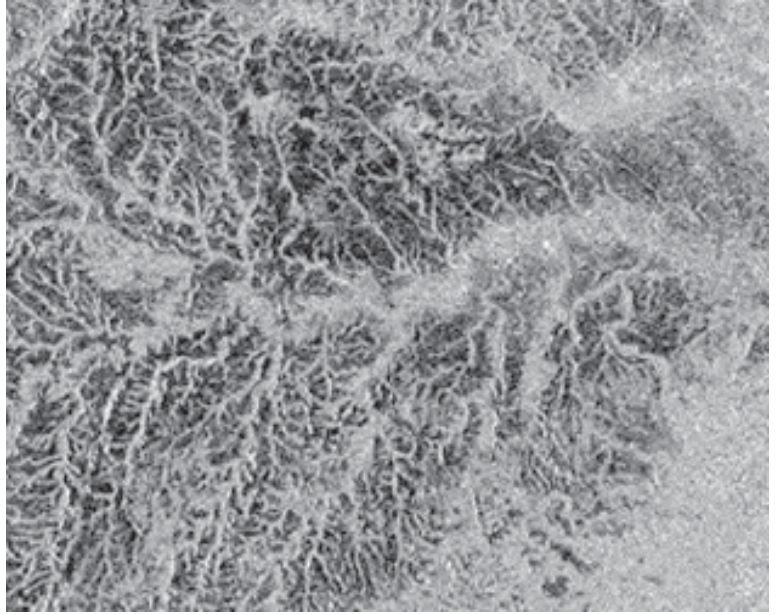

Fig. 5. Third component of the PCA of the deforested area of Antalaha. Black values are areas of deforestation while middle gray levels correspond to unchanged areas. 


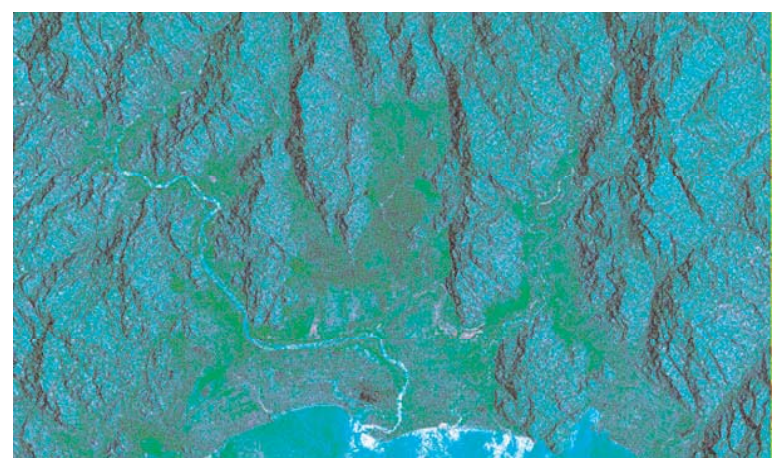

Fig. 6. False color composition of the three components of PCA of Maroentsetra (first component in blue, second component in green, third component in red). The yellow green (green + red) values can be attributed to changes between the two images.

All the methods above are generally helpful for visual interpretation of changes in remote sensing scenes. Yet, the presence of a speckle noise in ERS images remains an obstacle for the mapping and quantification of changes. There is accordingly a need to combine change detection techniques with filters for speckle reduction.

\section{FILTERS}

Many filtering techniques have been developed to remove the speckle in radar images while preserving both the radiometrical information and the spatial resolution of the initial image. Two types of approaches are commonly reported: the use of statistical filters such as the Lee Filter (Lee, 1981) where the existence of an additive or multiplicative noise is assumed (Fig. 7) or the use of morphological filters performing a smoothing of the gray-tone images thanks to the properties of such morphological transformations as erosion or dilation. Here, we rely on other filters belonging to the classes above. In what follow, the output images are presented in a binarized form (same threshold systematically applied) allowing a straightforward evaluation of the noise reduction.

\section{Statistical filter}

In this filter (Std filter), it is assumed the speckle noise is lower, or higher, than a range defined by the mean and the standard deviation of a square sliding window (Eq. 1). All pixels whose value is out of the local range are considered as noise and are replaced by the majority value (modal value) of the sliding window; all pixels whose value falls within the admissible range do not change. This statistical filter leads to a reduction of the noisy aspect of the original image, without loose of the radiometrical content or the spatial resolution (Fig. 8). It can be applied straightforwardly to time series images for multitemporal speckle reduction. In the latter case, an average mean and standard deviation of the series of images are compared to the pixels of one image.

$$
Y_{i j}=X_{i j} \text { if } \alpha_{k}<X_{i j}<\beta_{k} \text { else } Y_{i j}=M_{k} \text {. }
$$

Where $\alpha_{k}=x_{k}-\sigma_{k}, \beta_{k}=x_{k}+\sigma_{k}$ and $M_{k}$ is the majority value or modal value, $\mathrm{x}_{\mathrm{k}}$ is the average value and $\sigma_{\mathrm{k}}$ is the standard deviation of a sliding window of size $\mathrm{k}$.

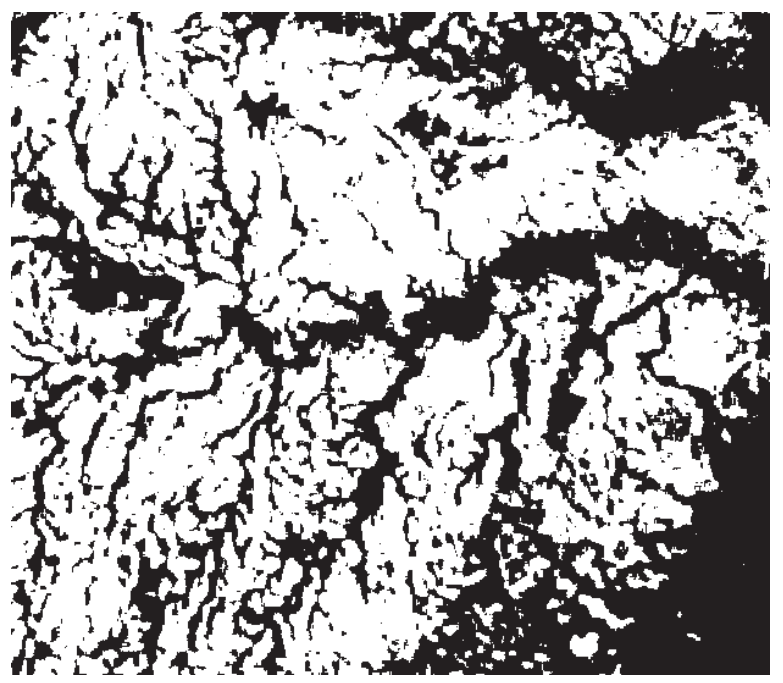

Fig. 7. Threshold Lee filter of the third component displayed in Fig. 5. This filter, commonly used in noise reduction tends however to deteriorate the spatial resolution.

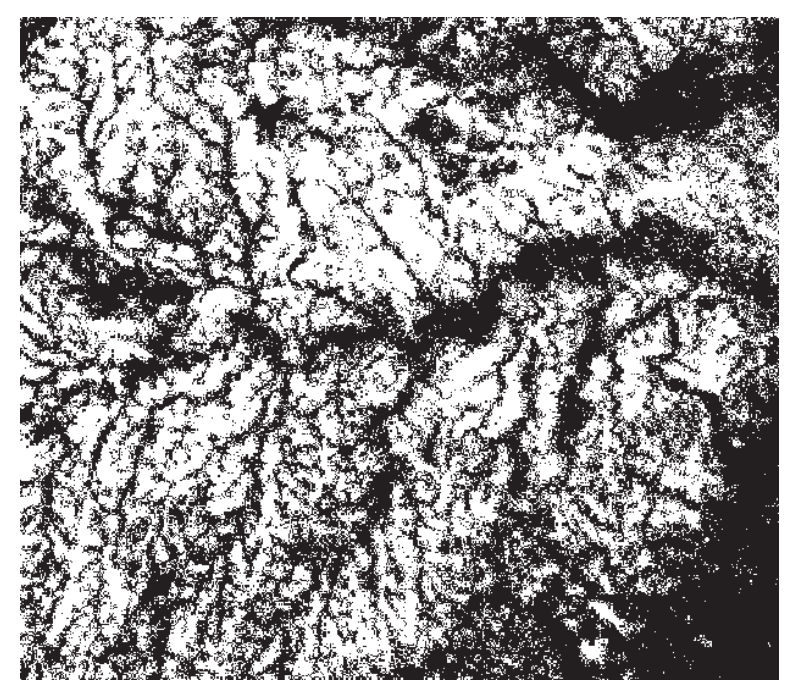

Fig. 8. Threshold statistical filter on the third component showing the speckle reduction and the preservation of the morphology and spatial resolution.

\section{Morphological filters}

Self-duality is one of the properties required for gray-tone image smoothing. Serra (1988) noticed that the median filter is self-dual, but not idempotent. Relying on morphological methods, it is possible to build filters having both properties (Serra, 1988): in 
the case of a complete lattice, the morphological center $\beta$ between an overfilter ( $\vee$-filter) $f$ and an underfilter $(\wedge$-filter $) g$ is expressed as:

$$
\beta=(I \wedge f) \vee g=(I \vee g) \wedge f .
$$

Given a morphological opening $\gamma$ (with a convex structural element $B$ ) and the dual morphological closing $\varphi$, the center $\beta$ is constructed with $f=\varphi \gamma \varphi$ and $\mathrm{g}=\gamma \varphi \gamma$. On the one hand, this first filter is quite efficient for smoothing gray-tone image smoothing. On the other hand, it is based on "classical" morphological opening and closing, and the morphology of the output image depends on the structural element size and shape. From this point of view, this filter does not preserve the morphology of the undestroyed elements.

It has been now frequently shown that digital connected filters produce gray-tone flat zones (Crespo et al., 1993). Moreover, filters based on a composition of connected openings and closings have the same algebraic properties than those based on openings and closings (Eq. 2).

Noting $\gamma_{c}$ the opening by reconstruction, and $\varphi_{c}$ the closing by reconstruction, we applied to the original radar image a filter $\beta \mathrm{c}$ representing the center of filters $f_{c}$ and $g_{c}$ and defined as:

$$
\beta_{c}=\left(I \wedge f_{c}\right) \vee g_{c}
$$

where $f_{c}=\varphi_{c} \gamma_{c} \varphi_{c}$ and $g_{c}=\gamma_{c} \varphi_{c} \gamma_{c}$.

The $\beta_{\mathrm{c}}$ filter (Mering and Parrot 1994) is applied here to a subzone of the ERS-1 scene of the deforested area of Madagascar (Figs. 5 and 9). On the resulting image (Fig. 9), the speckle has been partially removed and the contours of the structures have been restored.

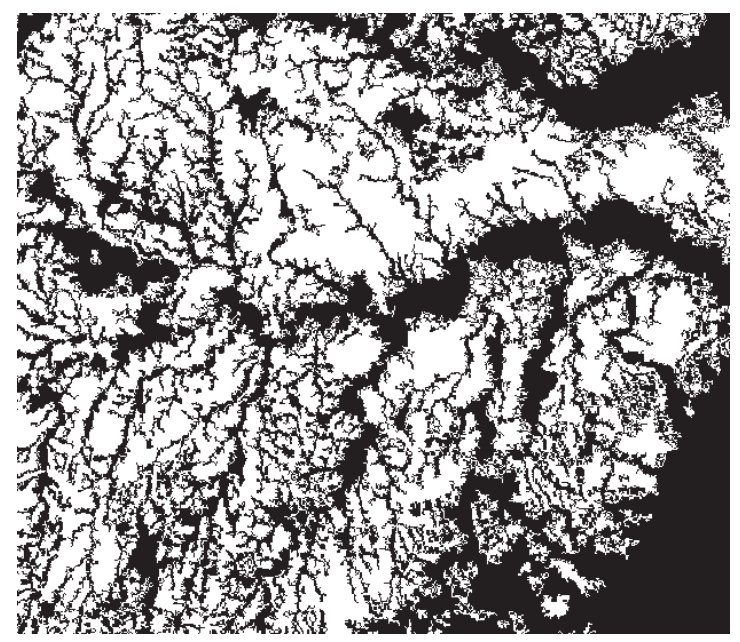

Fig. 9. Threshold connected center filter of the third component displayed in Fig. 5. With this filter we can see that the morphology is preserved while speckle noise is reduced.

\section{RESULTS AND DISCUSSION}

To estimate the efficiency of each filter with respect to noise reduction and morphology conservation, we threshold (in the same conditions) all filtered images and performed a granulometric analysis of the resulting binary images. Fig. 10 shows the input image to the filters (third component computing from an ACP of two ERS SAR images and an image of ratio) after thresholding. In the binarized Fig. 10, we can observe the noisy aspect of the original image characterized by many small elements of size one or two pixels. Fig. 8 shows the threshold Std filter of the original image (Fig. 5). Many small objects have disappeared, but we can still observe some of them. The morphology of the main objects is close to the original morphology. The image processed with the connected center filter is shown in Fig. 9. This filter gives good results in terms of speckle noise reduction and morphology preservation. The granulometric analysis (Fig. 11) shows that the number of elements of size 1 and 2 pixels is five times less in the connected-centerfiltered image than in the original one. In the Std filtered image, elements of the same size (1 and 2 pixels) are only three times less numerous that in the original image. The Lee filter (Fig. 7) suppresses almost all elements of size 1 and 2. Yet, we can observe in Fig. 7 that it does not preserve the morphology of larger objects.

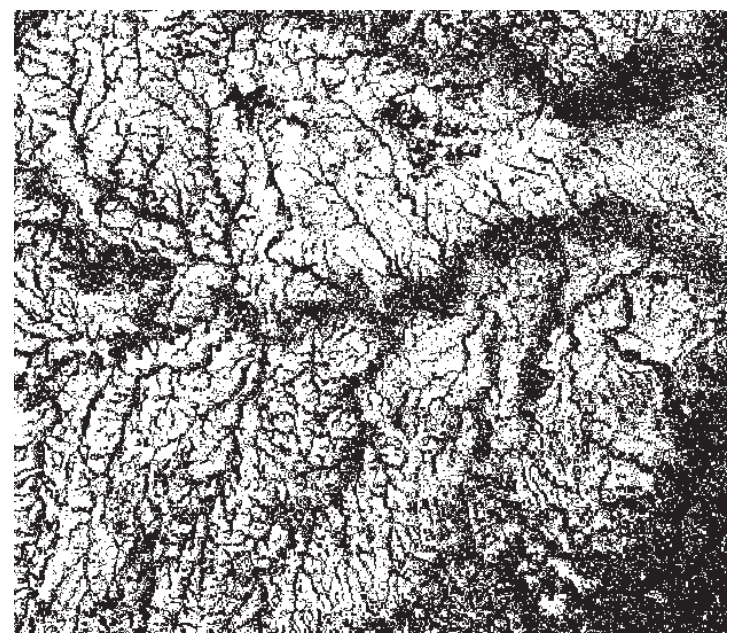

Fig. 10. Threshold version of the original image displayed in Fig. 5. In this image, the noisy aspect due to the speckle is clearly visible.

We also made four radiometric profiles in the gray level outputs of the different filters (Fig. 12). Those profiles show that the Std filter and the connected center filter preserve more the original morphology of the object than the Lee filter producing a 
very smoothed aspect. One will note in Fig. 12 that the connected center filter profile is the closest to the original profile.

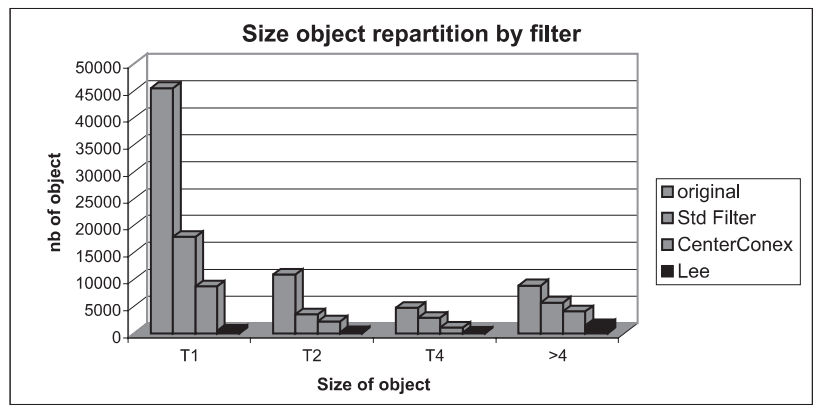

Fig. 11. Granulometric distribution of 4 sizes of objects in filtered images of an ERS SAR image on the Madagascar. Elements of size 1 by 1 pixel are more filtered by all filters than elements of larger sizes.

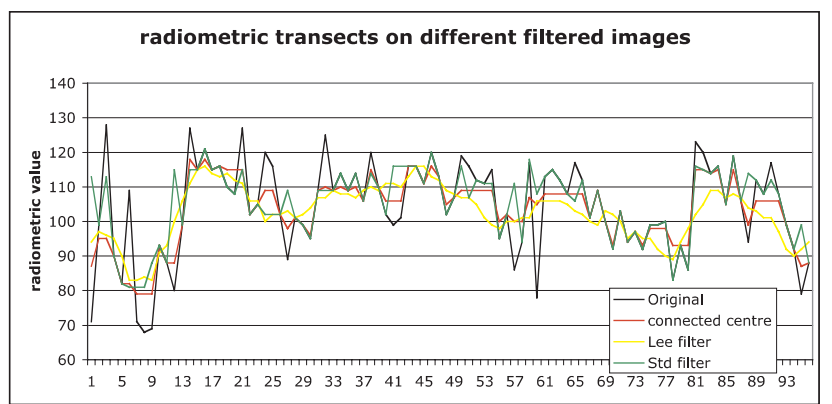

Fig. 12. Radiometric profiles of different ERS SAR filtered images. We can see that the Std filter and the connected center filter preserve the local morphology recalled by the original profile. The Lee filter keeps the trend of the original curve, but destroys the local morphology.
The Std filter preserves the spatial resolution of the image and can be applied to a series of multitemporal images (Fig. 8). It also preserves the edges of the structure.

Morphological filters, such as connected center filter, are more efficient to reduce speckle noise and to preserve the edges of structures thanks to the reconstruction procedures.

\section{REFERENCES}

Crespo J, Serra J, Schafer RW (1993). Image Segmentation using Connected Filters. In: Serra and Salembier, eds. Mathematical Morphology and its Applications to Signal Processing, 52-7.

Lee JS (1981). Refined filtering of image noise using local statistics. Computer Graphic and Image Processing 15:380-9.

Mering C, Parrot JF (1994). Radar image analysis using morphological filters. In: Serra and Soille, eds. Mathematical Morphology and its Applications to Signal Processing, 353-60.

Gong P (1993). Change detection using principal component analysis. Canadian Journal of Remote Sensing 19:22-9.

Serra J (1988). Image Analysis and Mathematical Morphology, vol. 2. Theoritical Advances. London: Academic Press.

Singh A (1989). Digital change detection techniques using remotely sensed data. In. Journal of Remote Sensing 10:989-1003. 